\title{
Bladder neck disease and kidney damage
}

\author{
Anna Mudoni ${ }^{1}$, Francesco Caccetta ${ }^{1}$, Maurizio Caroppo ${ }^{1}$, Fernando Musio ${ }^{1}$, Antonella Accogli ${ }^{1}$, \\ Maria Dolores Zacheo ${ }^{1}$, Domenica Maria Lucia Burzo ${ }^{1}$, Giancamillo Carluccio ${ }^{2}$, Vitale Nuzzo ${ }^{1}$ \\ ${ }^{1}$ Department of Nephrology and Dialysis, "Cardinale G. Panico" Hospital, Tricase (Lecce), Italy; \\ 2 Department of Urology "Cardinale G. Panico" Hospital, Tricase (Lecce), Italy.
}

\begin{abstract}
Summary Primary bladder neck obstruction (PBNO) was first described in men by Marion in 1933. The precise cause of PBNO has not been clearly elucidated. This paper review the theories on etiology, clinical presentation, diagnostic evaluation and treatments for PBNO. Also this paper focuses on management of patients with complications like acute urine retention, hydroureteronephrosis and severe renal failure. The treatment options for men and women with PBNO include careful clinical evaluation, pharmacotherapy with alpha-blockers and surgical intervention.
\end{abstract}

KEY WORDS: Primary bladder neck obstruction; Acute urine retention; Renal failure; Ultrasound; Videourodynamics; Alpha-blockers.

Submitted 3 October 2014; Accepted 31 October 2014

\section{INTRODUCTION}

The bladder neck disease is a cervico-urethral obstruction that results in an obstructed flow of urine due to the incomplete opening of the bladder neck during urination.

To date, the etiopathogenesis is still unclear and may be given to an abnormality in the development of the bladder neck and the detrusor musculature, whereby the bladder neck, during urination, remains contracted (1-4).

The present paper focuses on PBNO complicated by acute urine retention and severe renal failure, treated with surgery and shows clinical, laboratory and instrumental long-term follow-up.

\section{Case report}

We describe the clinical history of a 48 years old male, sent to our attention from emergency room where he presented for marked asthenia, general malaise, and oliguria. His history was positive for nocturnal occasional urinary incontinence from childhood. For this reason he had been treated with alpha blockers for an unspecified time. Since 38 years he had not carried out clinical, chemistry, and instrumental inspection and he had sus- pended, for his own initiative, any type of medical treatment. The physical examination revealed: blood pressure 130/80 mmHg, rhythmic heart rate $105 \mathrm{bpm}$, breaks free, no lung stasis nor peripheral edema. The skin was pale and there was no signs of fluid overload. In the suprapubic area there was a hard mass, tense, sore from probable globe bladder. The diuresis was present.

The laboratory tests showed a mild anemia (hematocrit 31.9 vol.\%, hemoglobin $11.4 \mathrm{~g} / \mathrm{dl}$ ) and a severe renal impairment (blood urea $174 \mathrm{mg} / \mathrm{dl}$, s-creatinine 7.4 $\mathrm{mg} / \mathrm{dl}$ ). Blood gas analysis revealed metabolic acidosis ( $\mathrm{pH}$ 7.3 , bicarbonate $18 \mathrm{mmol} / \mathrm{L}, \mathrm{BE}$ ecf-5.6 mmol/L).

Serum electrolytes were normal. Erythrocyte sedimentation rate was $24 \mathrm{~mm}$, and urine analysis showed a density of 1010, pH 5.0, hemoglobin $0.10 \mathrm{mg} / \mathrm{dl}$, absence of protein and some erythrocytes in the sediment.

Urineculture was negative. There were not bleeding disorders, and liver and thyroid function were normal. The electrocardiogram highlighted sinus rhythm, tachycardia. The chest X-ray was negative. Abdominal ultrasound, performed at the admission to our Department of Nephrology, showed an increase in right kidney size (Figure 1), with a $138 \mathrm{~mm}$ longitudinal diameter (LD), and a normal size left kidney (LD $103 \mathrm{~mm}$ ).

The parenchymal thickness was bilaterally slightly reduced with bilateral severe hydroureteronephrosis.

The bladder appeared very relaxed, with thickened walls, jagged mucous design and there were presence of large particulate matter in suspension (estimated volume of $4500 \mathrm{ml}$ ) (Figure 2).

The urologist consulted, after an unsuccessful attempt to bladder catheterization, placed suprapubic transcutaneous Foley catheter, resulting an abundant outflow of urine hyperpigmentated.

The patient underwent a hydration therapy with sodium bicarbonate and polysaline solution, and antibiotic therapy with levofloxacin $500 \mathrm{mg} /$ day was started.

During hospitalization, indices of renal function gradually improved, as shown in Figure 3, with a correction of acid-base balance.

In the following days, the patient was submitted to retrograde and voiding cystourethrography that showed the bladder retrogradely filled with finely serrated walls. In the voiding phase only a thread-like opacification of the 


\section{Figure 1.}

Bilateral severe hydroureteronephrosis, right kidney increased size (LD $138 \mathrm{~mm}$ ), left kidney normal size (LD $103 \mathrm{~mm}$ ).

The parenchymal thickness bilaterally slightly reduced.

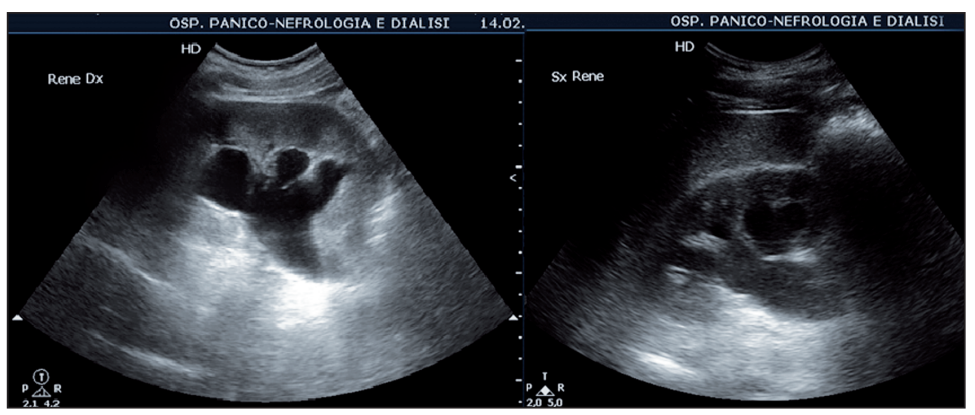

Figure 2.

Bladder over dilated at diagnosis with thickened walls (left); post-voiding residual volume after treatment (about $100 \mathrm{ml}$ ) (right).

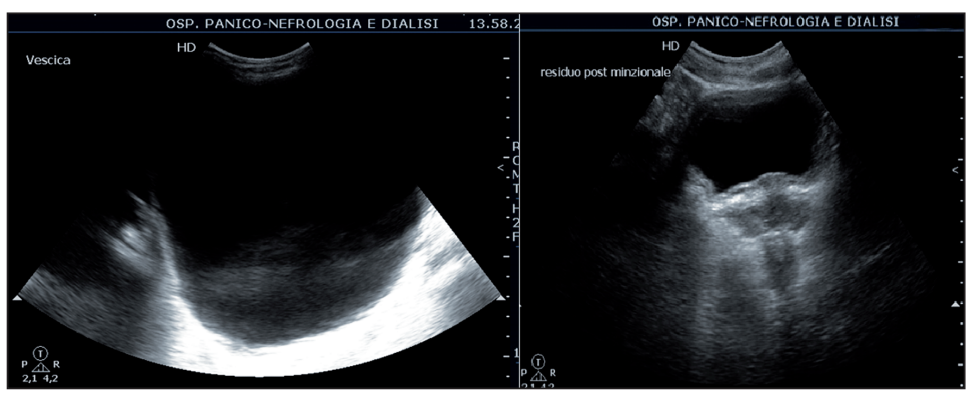

The sequential renal scintigraphy showed the ability of glomerular filtration rate less than the norm, with functional prevalence of the right kidney and absence of signs of obstructive disease.

Uroflowmetry revealed flow values and flow control in the standard curve.

Introduction, Discussion, Conclusions and other Figures are posted in Supplementary Materials in www.aiua.it

\section{REFERENCES}

1. Marion G. Surgery of the neck of the bladder. $\mathrm{Br} J$ Urol. 1933; 5:351-357.

2. Leadbetter GW, Leadbetter WF. Diagnosis and treatment of congenital bladder neck obstruction in children N Engl. J Med.1959; 260:633.

3. Nitti VW. Primary bladder neck obstruction in men and women. Rev Urol. 2005; 7 Suppl 8:S12-7.

4. Padmanabhan P, Nitti VW. Primary bladder neck obstruction in men,women, and children. Curr Urol Rep. 2007; 8:379-84. urethra was appreciated, whereas, in the retrograde opacification, signals stricture of the membranous urethra was detected.

Subsequently, in general anesthesia, he was submitted to urethrocystoscopy with evidence of obstructive bladder neck. Endoscopic incision of the bladder neck by laser fiber was performed with urinary catheter placement and subsequent removal of the suprapubic Foley catheter. The postoperative course was uneventful, so the urinary catheter was removed and a valid resumption of spontaneous voiding occurred.

At discharge the patient was in good general condition, with valid urination of normochromic urine and improvement in renal function (s-creatinine $2.5 \mathrm{mg} / \mathrm{dl}$, cGFR $27 \mathrm{ml} / \mathrm{min} / \mathrm{m}^{2} \mathrm{MDRD}$ formula).

The patient underwent a regular clinical, laboratory and instrumental follow-up (renal and bladder ultrasonography, uroflowmetry, renal scintigraphy) and, after more than a year, resolution of symptoms, absence of urinary tract infections and improvement in renal function (s-creatinine $1.64 \mathrm{mg} / \mathrm{dl}$, cGFR $88 \mathrm{ml} / \mathrm{min} / \mathrm{m}^{2}$ ) were observed. Sonographically, the right kidney presented a normal size (LD $108 \mathrm{~mm}$ ), regular shape, normal cortico-medullary thickness, resistive intrarenal index (RI) equal to 0.6 and pyelo-caliceal cavity not dilated without stone. Left kidney presented a small size (LD $81 \mathrm{~mm}$ ), regular shape, thickness cortico-medullary reduced, (RI 0.9), with a II-III degree hydronephrosis, without stones (Figure 4). Bladder showed thickened walls and a post-voiding residue was present (about $100 \mathrm{ml}$ ) (Figure 2).
Correspondence

Anna Mudoni, MD (Corresponding Author)

mudonia@libero.it

Francesco Caccetta, MD

Maurizio Caroppo, MD

Fernando Musio, MD

Antonella Accogli, MD

Maria Dolores Zacheo, MD

Domenica Maria Lucia Burzo, MD

Vitale Nuzzo, MD

Department of Nephrology and Dialysis, "Cardinale G. Panico" Hospital Via S. Pio X 4, 73039 Tricase (Lecce), Italy

Giancamillo Carluccio, MD

Department of Urology, "Cardinale G. Panico" Hospital

Via S. Pio X 4 - 73039 Tricase (Lecce), Italy 